COMMENT. Evaluation of neuropsychological function is an important aspect of epilepsy management in children. It is disturbing to find that academic difficulties may antedate the onset of epilepsy and can be associated with significant reductions in grey matter, specifically in the left occipital and parietal lobes. The mechanisms of these epilepsy related neuropsychological and cerebral volume disorders remain to be determined. The seizures per se and/or adverse effects of antiepileptic medications are not the only factors responsible for academic difficulties among children with epilepsy, and preexisting developmental cerebral abnormalities may be more important causes.

\title{
HIPPOCAMPAL ABNORMALITIES IN PROLONGED FEBRILE SEIZURES
}

Apparent diffusion coefficient (ADC) measurements were used to characterize hippocampal edema within 5 days of a prolonged febrile seizure (PFS) in a study at Great Ormond Street Hospital, London, UK. A reduction in ADC between acute and 4-8 month follow-up measurements was indicative of early vasogenic edema, followed by recovery in children investigated within 2 days, but not in those measured between 3 and 5 days of a PFS. An expected age dependence decrease in ADC observed in control subjects was not present in children following a PFS. The findings are consistent with resolution of an early onset acute vasogenic edema that follows a PFS. The authors propose that the ADC data reflect a preexisting developmental hippocampal abnormality that predisposes to a PFS. The data also suggest that the vasogenic edema developing within 2 days after a PFS resolves in 3-5 days. (Scott RC, King MD, Gadian DG, Neville BGR, Connelly A. Prolonged febrile seizures are associated with hippocampal vasogenic edema and developmental change. Epilpsia Sept 2006;47:1493-1498). (Reprints: Dr Rod C Scott, The Wolfson Centre, Mecklenburgh Square, London WC1N 2AP, UK).

COMMENT. These ADC findings corroborate the longitudinal MRI data reported by these authors previously (Scott RC et al. Brain 2003;126:2551-2557). Within $48 \mathrm{~h}$ of a PFC hippocampal volumes were enlarged and T2 relaxation times prolonged, whereas MRI studies delayed $>48 \mathrm{~h}$ but within 5 days of PFC revealed large hippocampal volumes and normal T2 relaxation time. These findings were suggestive of hippocampal edema that is resolving within 5 days of a PFC. Repeat MRI at 4-8 month follow-up showed reduction in hippocampal volume with asymmetry and reduced T2 relaxation time compared to the first exam. It is postulated that mesial temporal sclerosis may develop after a lag period, or the hippocampal asymmetry represents a return to a preexisting hippocampal developmental abnormalty that antedates the PFC. (Ped Neur Briefs Nov 2003;17:83).

Hippocampal asymmetry in 6-year follow-up MRI study of complicated convulsion at University of Sheffield, UK. (Farrow TFD et al. Pediatr Neurol 2006;35:257-260). Significant hippocampal asymmetry unrelated to edema was initially reported within 2 weeks of a first complicated early childhood convulsion in $>50 \%$ of 17 subjects tested. At 6-year follow-up, 3 of 8 retested showed significantly greater asymmetry and 2, a modest increase in asymmetry; 2 showed no change and 1 a resolution of asymmetry. The asymmetry was detected only by volumetric analysis of MRI and relaxation times, and was consistent with a progressive pathology and developing hippocampal sclerosis in 2 patients. 
Frontal subcortial white matter lesions following PFS with encephalopathy are described in a diffusion-weighted MRI study between 3 and 9 days after the seizure. Diffusion abnormalities disappeared between days 9 and 25, leaving cerebral atrophy after 2 weeks (Takanashi J et al. Neurology 2006;66:1304-1309; Ped Neur Briefs May 2006;20:33).

\section{ETIOLOGY, EEG, AND MRI FINDINGS IN NONCONVULSIVE SEIZURES WITH IMPAIRED CONSCIOUSNESS}

Nonconvulsive seizures (NCS) in children admitted to the Pediatric Intensive Care Unit (PICU) from Jan 2000 to Dec 2003 were analyzed in a study at The Hospital for Sick Children, Toronto, Ont, Canada. A NCS was defined as a deterioration of consciousness accompanied by at least one episode of electrographic seizure lasting longer than $10 \mathrm{~s}$. Of 141 patients admitted with unexplained altered consciousness, no overt seizure, and EEG recording within $24 \mathrm{~h}, 23(16.3 \%)$ had NCS. Male to female ratio was 1.9:1. The mean age was 40.1 mo (range: 1 mo to $18 \mathrm{y}$ ); infants $<1$ mo were excluded. No preexisting disease was present in $10(43 \%) ; 8(35 \%)$ had a history of seizures, $2(9 \%)$ congenital heart disease, and 3 $(13 \%)$ other underlying disease. None had nonconvulsive status epilepticus. The etiology of NCS was an acute structural brain lesion in 11 (48\%); cerebral infarction in 6, subdural hematoma in 3, cerebral hemorrhage in 1, and AV malformation in 1. Other etiologies included meningitis in $3(13 \%)$, seizure disorder in $3(13 \%)$, sepsis in 2 and pneumonia in 2. EEG showed right-sided epileptiform discharges in $9(39.2 \%)$, most commonly temporal; the EEG pattern was S/W in $9(39.1 \%)$. The mean duration of EEG discharge was $159 \mathrm{~s}$ (range: $10 \mathrm{~s}$ to $11 \mathrm{~min})$. MRI or CT was abnormal in $18(78.2 \%)$, most commonly cerebral infarction in $6(26.1 \%)$, followed by diffuse edema in $5(21.7 \%)$. Brain imaging and EEG were concordant in localization in $10(55.6 \%)$. All seizures responded to treatment with IV phenobarbital or phenytoin. (Saengpattrachai M, Sharma R, Hunjan A et al. Nonconvulsive seizures in the pediatric intensive care unit: Etiology, EEG, and brain imaging findings. Epilepsia Sept 2006;47:1510-1518). (Reprints: Dr MA Cortez, Division Neurology, Department of Pediatrics, The Hospital for Sick Children, 555 University Ave, Toronto, Ontario M5G 1X8, Canada).

COMMENT. Of children admitted to the PICU with altered state of consciousness, $16 \%$ have NCS. A higher prevalence (33\%) reported in a previous study is explained by the inclusion of neonates and PLEDs. In contrast to adults who often have preexisting epilepsy and traumatic brain injury as precursors of NCS, children have no preexisting disease in $43 \%$ of cases, cerebral infarction as the most common NCS etiology, and a predominance of right hemisphere localization. An early EEG is indicated in children with deterioration of consciousness of uncertain origin.

Nonconvulsive status epilepticus is described in 19 pediatric patients admitted to the Epilepsy Center, Columbia University, NY, June 2000 - Dec 2003. Five developed NCSE following convulsive SE and 12 followed brief convulsions. Acute hypoxic-ischemic injury was the most frequent etiology (in $5(26 \%)$ ). Six had associated periodic lateralized epileptiform discharges (PLEDs), and 1 had generalized periodic epileptiform discharges (GPEDs), both associated with worse outcome. Five $(26 \%)$ died of the underlying acute 